\title{
II controllo di gestione e la qualità in sanità
}

\author{
G. Quintaliani ${ }^{\text {1, S. Cencetti }}{ }^{2}$ \\ ${ }^{1}$ Controllo di Gestione Azienda Ospedale di Perugia, Perugia \\ ${ }^{2}$ Direzione Sanitaria Ospedale Policlinico, Roma
}

fronte della aziendalizzazione della sanità, l'approccio economico del settore industriale e commerciale non può essere trasferito, senza opportuni accorgimenti, al settore sanitario.

In sanità l'obiettivo finale non è il profitto ma è l'ottenimento di risultati in termini di salute.

Inoltre l'aspetto sanitario si differenzia da altri campi poiché in sanità non è il cliente che condiziona il mercato con i suoi gesti, le sue preferenze, le sue esigenze, ma è il medico il più importante decisore della spesa.

La qualità professionale e, quindi, l'appropriatezza diventa il cardine per gestire adeguatamente ("qualità gestionale") il servizio e le prestazioni sanitarie in favore dei cittadini utenti nei cui riguardi va posta tutta l'attenzione possibile in rapporto alle loro esigenze, alle loro aspettative ("qualità percepita"), da correlare alle peculiarità del settore.

A sua volta appropriatezza significa fare le cose giuste quando servono sia nel caso in cui si affrontino gli aspetti organizzativi, amministrativi, educativo-formativi, sia i sanitari (preventivi, curativi, riabilitativi); per decidere della appropriatezza ci si ri- ferisce a ciò che è dimostrato e riconosciuto.

Il "manager" che vuole operare in campo sanitario con un minimo di efficacia deve tenere conto del fatto che i costi e le strutture dipendono dal volume di attività e dal consumo e che la loro adeguatezza alle necessità e ai risultati in termini di qualità e quantità di vita, sono il fondamento e l'obiettivo stesso di tutto il settore sanitario.

Non solo per il manager, ma per tutti coloro che hanno responsabilità di programmazione, organizzazione e gestione dei servizi sanitari, ad iniziare dai medici, si pone quindi una sfida, per certi versi paradossale: migliorare la quantità e la qualità professionale e contenere i costi.

\section{Il controllo di gestione}

Il controllo di gestione costituisce elemento elettivo per rispondere a questa sfida, in quanto consente alla dirigenza aziendale di conoscere e monitorare consumi, strategie e risultati raggiunti in relazione agli obiettivi di politica sanitaria aziendale prestabiliti.

Si può definire come un insieme coordinato e integrato di criteri, strumenti e procedure finalizzati ad attivare un processo iterativo di programmazione degli obiettivi da conseguire e di verifica della rispondenza dell'andamento gestionale rispetto agli obiettivi prefissati, al fine di consentire a una organizzazione di conseguire i propri obiettivi con la massima efficacia e con la massima efficienza possibile (contenuti economico-gestionali) coniugandoli con contenuti di carattere tecnicoprofessionale, che orientino e supportino la gestione in una logica di qualità totale.

Per ottenere questo risultato bisogna tener conto di alcune considerazioni:

a) l'informazione è potere a tutti i livelli e quindi non deve essere demonizzata da parte di chi si sente controllato (il singolo medico o Referente del Centro di Responsabilità) ma deve anzi essere accettata e controllata;

b) ne discende che per poter essere efficace un sistema deve prevedere dei responsabili di centri di costo che rispondono al Referente del Centro di Responsabilità, ma che devono essere ben consci di poter e dover contare su risorse a loro affidate e sul cui uso saranno chia- 
mati a rispondere in relazione agli obiettivi assegnati;

c) che potrebbe essere auspicabile passare alla responsabilità del caso trattato piuttosto che alla responsabilità del modello organizzativo.

Il controllo di gestione non è quindi un mero fatto contabile né è sinonimo di contabilità dei costi; è invece un processo direzionale.

Il controllo di gestione è in sintesi l'insieme combinato e coordinato di tre tecniche aziendali:

- un sistema previsionale di budgeting in cui la Direzione stabilisce gli obiettivi generali e le risorse concordando con i Referenti del Centro di Responsabilità i modi, i tempi e le risorse da impiegare per raggiungerli (il budget è sempre un processo legato a un tempo stabilito: semestri o anni). In questa fase sarebbe opportuno che si concordassero anche tra Dirigenza e Centro di Responsabilità gli indicatori e gli standard che detteranno POI i risultati:

- un sistema di rilevazione corrente dei fenomeni aziendali, denominato contabilità analitica che si deve avvalere di un sistema informativo di un certo impegno sia economico che strutturale;

- un sistema di reporting, atto a coniugare tramite indicatori di sintesi i dati dei due momenti precedenti, effettuando una vera e concreta attività di valutazione.

La responsabilità dell'intero processo è del Direttore Generale, momento di sintesi del processo decisionale, che si avvale di apposite articolazioni aziendali, costituenti l'Ufficio programmazione e controllo di gestione ma è necessario che si passi attraverso la responsabilizzazione e il coinvolgimento dei medici.

\section{Il medico e il controllo di gestione}

Le politiche puramente economicistiche di contenimento della spesa sinora adottate si sono tutte caratterizzate per una connotazione comune: il fatto di essere strumenti di controllo esterno alle dinamiche di effettiva generazione della spesa (Regione, Stato ecc.).

Il problema di fondo è quello di ricomporre in un unico momento decisionale la responsabilità tecnico-professionale e la responsabilità economico-gestionale e di pervenire a una gestione programmata delle attività sanitarie, attraverso la preliminare fissazione di un budget di esercizio, inteso quale specificazione di obiettivi-attività-risorse organicamente integrati in un programma di attività.

Non si deve intendere l' adozione del processo di controllo di gestione come la trasformazione integrale del medico in manager; si auspica una presa di coscienza, da parte del medico, delle implicazioni economiche delle decisioni assunte sul piano diagnostico terapeutico e sul piano delle modalità tecnico-organizzative.

In sostanza l'azione sanitaria diventa efficace se inserita in un contesto di sistema nell'ambito del quale ciascun soggetto coinvolto svolge il proprio compito in una logica integrata e nell'ambito di una chiarezza di rapporti. Il ruolo del medico, quindi, non può essere limitato alla semplice fetta di processo che lo vede attore principale, ma deve essere tale da prendere in considerazione il successo finale della sua azione.

La forte responsabilizzazione verso l'equilibrio economico finanziario sia rispetto alla singola azienda sia rispetto al sistema regionale nel quale essa è inserita porteranno progressivamente il sistema a effettuare due valutazioni fondamentali:

1) la valutazione di quali bisogni debbano essere soddisfatti. La quantità di risorse disponibili e la rigidità determinata dall'equilibrio di bilancio non consente più di garantire risposte rispetto a qualunque tipologia di bisogno;

2) la valutazione dell' efficacia della prestazione erogata. In altri termini si porrà il problema di chi può concretamente e in modo efficace ed efficiente garantire una risposta a uno specifico bisogno; non si porrà quindi solo il problema di stabilire che una determinata patologia rientra nei livelli di assistenza uniformi, ma che la risposta a quel bisogno può essere garantita solo da determinate strutture.

\section{Il problema della qualità}

Risulta che, nello scenario delineato, si rivelerà vincente chi si preoccuperà di affrontare in modo concreto $\mathrm{e}$ sistematico il problema della qualità. Questi ultimi si troveranno nella condizione di governare il sistema piuttosto che di essere gestiti da esso.

L'attivazione di un programma di qualità non si basa solo sulla definizione delle "buone pratiche", tradotte in termini di procedure, protocolli, percorsi e nella definizione di parametri di riferimento per la misurazione della qualità ma è comunque fondamentale l' introduzione dei percorsi diagnostico-terapeutici che possano garantire una serie di opportunità di tutto rilievo:

- misurare il tipo e la quantità delle prestazioni effettuate e, conseguentemente, di valutarne l'opportunità sia sul piano clinico che economico; - valutare le modalità con le quali le diverse prestazioni si sono susseguite rispetto al percorso complessivo del paziente e quindi di individuare possibili alternative più efficaci e/o efficienti;

- estrapolare una serie di informazioni non monetarie di fondamentale importanza nella gestione operativa della singola unità di produzione;

- possibilità, rispetto a patologie semplici e a fronte di volumi di produzione statisticamente significativi, di elaborare dei "percorsi tipo" o orientativi con i quali attivare un continuo confronto con i percorsi reali sia al fine di comprendere le cause degli eventuali scostamenti che di ridefinizione "in progress" dei "percorsi tipo" e alternativi (day surgery, pacchetti ambulatoriali, assistenza a domicilio ecc.).

\section{Lo sviluppo dei percorsi diagnostico-terapeutici}

È indubbio comunque che al di là delle accezioni astratte la qualità della sanità e della medicina si risolve, alla fine di tutto il processo, nella qualità del singolo atto medico e 
quindi la perizia e le decisioni circa i mezzi da utilizzare sono fortemente condizionati dalla conoscenza scientifica e dalle capacità tecniche dell'operatore che si trova a prendere decisioni riguardo il singolo paziente. Ed è inoltre ovvio che, seppure un coordinamento adeguato aumenti le probabilità che risorse assistenziali di qualità appropriata si trovino nella quantità necessaria e al momento giusto dove se ne ravveda la necessità, è altresì vero che il loro corretto ed efficace utilizzo dipenderà solo dalla capacità dell'operatore.

A sua volta la capacità di risolvere in modo efficace i problemi dei pazienti che richiedono intervento sanitario dipende sostanzialmente da due fattori principali:

a) la chiara definizione dell'insieme delle azioni da intraprendere rispetto al caso da trattare e della loro migliore sequenza sul piano temporale;

b) la possibilità di disporre, da parte del medico responsabile della gestione del caso, degli apporti necessari, al momento più idoneo, forniti dagli altri soggetti coinvolti nella gestione del paziente.

La definizione dei percorsi diagnostico-terapeutici non è un' operazione volta a annullare o limitare l'autonomia clinica dei singoli professionisti ma, al contrario, è uno strumento messo a disposizione e gestito dal singolo professionista responsabile del caso, finalizzato a:

1) fornire il miglior servizio possibile al paziente anche attraverso la comunicazione a lui e ai familiari di quanto probabilmente lo attende;

2) migliorare i rapporti di collaborazione e di scambio interni ed esterni all'organizzazione;

3)valorizzare il ruolo professionale degli operatori, in primo luogo quello medico, con riferimento al caso trattato nella prospettiva dello sviluppo della figura dei "case manager".

La lettura dei dati dei percorsi effettivi, confrontati anche con quanto elaborato dalla letteratura, consente di comprendere e valutare le diverse pratiche seguite e le relazioni interne ed esterne instaurate, così da attivare un sistematico processo di apprendi- mento organizzativo a livello di singolo, di gruppo e d'azienda.

Il termine Percorso Diagnostico-Terapeutico, in considerazione della definizione formulata, non deve essere confuso con il concetto di Linea Guida. Quest' ultima, infatti, può essere identificata, anche se in realtà non viene mai formulata a un grado di dettaglio simile a quello utilizzato per descrivere i percorsi diagnosticoterapeutici, con una sorta di percorso ideale che prende in considerazione la tecnologia d'avanguardia e modelli organizzativi ottimali. Al contrario, il percorso diagnostico-terapeutico rappresenta la modalità possibile di trattamento di un paziente calata in un contesto aziendale specifico. La rilevazione e la riprogettazione dei percorsi diagnostico-terapeutici si inseriscono nel processo di controllo, revisione e miglioramento continuo della gestione e, quindi, della qualità dell'attività ospedaliera. Esso prende avvio proprio dalla rilevazione dell'insieme delle azioni compiute per pazienti con problematiche analoghe di salute e si estrinseca nell'individuazione della pratica ritenuta "più adeguata" dagli operatori e nell'effettuazione di confronti e valutazioni periodiche dei percorsi effettivamente compiuti in modo da generare la modifica dei comportamenti dei vari attori interessati (singoli professionisti, Centro di Responsabilità e i rapporti ospedale-territorio) oltreché l'aggiornamento della progettazione dei percorsi diagnostico-terapeutici. Con l'impiego dei percorsi diagnostico-terapeutici ci si propone di perseguire, quale articolazione delle finalità precedentemente enunciate, i seguenti obiettivi:

1) rispetto al paziente: a - disporre di un' ipotesi di lavoro, posta all' attenzione di tutti gli operatori interessati, frutto dell'esperienza e aggiornata sulla base delle più moderne conoscenze scientifiche; $b$ - coinvolgere il paziente attraverso l'informazione di quello che l'aspetta e di come lavora l'ospedale;

2) rispetto all'organizzazione: a - focalizzare, rispetto al singolo paziente e alla diagnosi di riferimento, il legame esistente fra qualità delle iniziati- ve/attività sanitarie e utilizzo delle risorse; $\mathrm{b}$ - individuare le discipline e $\mathrm{i}$ Centri di Responsabilità coinvolti al fine di definirne il ruolo nell'ambito dei trattamenti diagnostico-terapeutico; c - descrivere le risorse (durata prevedibile della degenza, esami, farmaci ecc.) necessarie a trattare un particolare gruppo di pazienti al fine di valutarne l'appropriatezza, la qualità e il valore economico (costo); d - contribuire all'obiettivo generale di migliorare continuamente la qualità e la resa economica dei servizi ai pazienti;

3) rispetto al ruolo professionale: a - contribuire con un metodo condiviso a sistematicizzare le pratiche e le metodologie sanitarie seguite; b fornire strumenti per la formazione e l'aggiornamento degli operatori; c elaborare linee di comportamento che tutelino l'azione del medico rispetto alle responsabilità giuridiche che possono derivare dall'inosservanza delle "buone pratiche"; d - sviluppare e valorizzare il ruolo degli operatori (case manager medico ed infermieristico) soprattutto di quelli direttamente impegnati nella gestione dei pazienti con la costituzione di appositi gruppi di lavoro.

Chiariti gli elementi di base che costituiscono il percorso diagnosticoterapeutico è possibile passare alla fase più operativa della esplicitazione di quest'ultimo che si caratterizza in tre momenti fondamentali:

1) definizione delle caratteristiche che determinano l'ingresso del paziente in un percorso. Si tratta pertanto di inquadrare, in termini generali, il problema di salute che si intende affrontare con l'attivazione del percorso e di stabilire quali parametri determinano l'ingresso del paziente nel percorso specifico;

2) definizione dell'insieme delle attività e della loro collocazione temporale rispetto all'intero percorso. Si tratta quindi di rappresentare, in modo puntuale, rispetto a uno schema che scandisce lo sviluppo temporale del trattamento del paziente, quali azioni si rendono necessarie e in quale momento al fine di perseguire uno specifico risultato; 
3) definizione del risultato atteso. $\mathrm{Si}$ tratta della fase più complessa e che richiede la possibilità di disporre di una base informativa statisticamente significativa. Essa consiste nello stabilire quale stato di salute ci si attende che il paziente abbia al termine del percorso. Esso può essere espresso da una combinazione di indicatori che si riferiscono alla vita residua attesa, al grado di autonomia recuperata, alla possibilità di reinserimento nella vita lavorativa ecc.

L'uso di protocolli comporta invariabilmente la definizione di strumenti che permettano di cogliere 1' adesione di una diagnosi al protocollo stesso $o$, in altri termini, la misurazione della qualità.

Ciò si ottiene definendo attentamente degli standard e dei criteri a cui attenersi.

I criteri possono essere definiti come definizioni quantitative che possono essere misurate anche al di fuori di un processo specifico tecnico (posti letto, numero di infermieri) o comunque sono espressione di un risultato clinico accettabile (valori della P.A. massima dopo una terapia farmacologica).

Gli standard sono invece i risultati attesi connessi ai criteri (2 infermieri ogni sei pazienti in dialisi, la P.A. dopo trattamento non deve superare i $140 \mathrm{~mm} / \mathrm{Hg} \mathrm{ecc}$ ).

È ovvio che solo delle società scientifiche sono in grado di elaborare dei criteri e degli standard a cui attenersi. Solo quando il mondo medico avrà elaborato dei protocolli attendibili, seri e che possano risolversi in una attuazione ragionevole e con costi accettabili si potrà superare questo dualismo tra componente amministrativo-burocratica e clinica.

Attualmente sono ben poche le società scientifiche in grado di formulare proposte attendibili; e se è vero che tale ritardo è soprattutto delle società scientifiche è anche del tutto vero che nella maggior parte dei casi i singoli Referenti del Centro di Responsabilità non sono stati in grado di elaborare, per le loro realtà, degli standard accettabili e ragionevoli rimanendo ancorati alla logica del tutto e del più dimenticando la regola fondamentale che il primo dovere di un responsabile di una struttura complessa non è fornire il massimo ma l'ottimo cercando di migliorare le condizioni in cui ci si trova ad operare.

In questo ambito la SIN ha svolto un lavoro encomiabile lungo due direttive:

a) organizzando le Linee Guida su un'ampia serie di problematiche e mettendole a disposizione su carta e su Internet;

b) nell'ambito della commissione Qualità e Accreditamento specificando molto chiaramente che sarebbe necessario e auspicabile che le singole unità di Nefrologia si dotino quanto più è possibile di regole scritte sull'osservanza delle varie procedure che possono essere richieste nello svolgimento del lavoro quotidiano.

\section{Conclusioni}

Il controllo di gestione è lo strumento fondamentale a disposizione della azienda e degli operatori per sapere cosa si fa, come lo si fa e perché lo si fa. La responsabilizzazione sul problema dei costi permetterà di effettuare scelte proficue sia per i pazienti sia per la collettività. È compito dei responsabili aziendali e dei Centri di Responsabilità avviare metodiche e procedure in grado di garantire la qualità. È necessario anche che i singoli medici, le loro organizzazioni interne ed esterne all'azienda combattano una battaglia che veda la qualità la primo posto; per ottenerla sarà necessario richiedere a gran voce sia strumenti tecnici (libri, riviste, collegamenti a Medline e a Internet), sia percorsi formativi (comandi, audit) che possano garantire un aggiornamento continuo e puntuale.

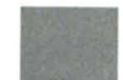

\section{BIBLIOGRAFIA}

1. Primicerio B, Tronci M. Total quality management in sanità. Ed. Luigi Pozzo 1998.

2. Bashshur RL. Telemedicine effects: cost, quality, and access. J Med Syst 1995; 19 (2): 81-91.

3. Brita-Rossi P, Adduci D, Kaufman J, Lipson SJ, Totte C, Wasserman K. Improving the process of care: the cost-quality value of interdisciplinary collaboration. J Nurs Care Qual 1996; 10 (2): 10-6.

4. Burton BA, Williams S. ESRD delivery models. Balancing quality and cost: views from the provider, insurer, and the outpatient setting. Nephrol News Issues 1995; 9 (6): 28.

5. Borgonovi E, et al. La programmazione ed il controllo di gestione nelle strutture sanitarie. McGraw Hill, Milano.

6. Zavattaro F. Il budget: una risposta alla crescita di complessità del sistema sanitario. Mecosan n 5 .

7. Casati G. Vallotti. Il collegamento tra sistema di budget e sistema di incentivazione nelle aziende ospedaliere: una possibile metodologia. Mecosan 1996; 17.

8. Casati G. Il percorso del paziente. Cergas 1999.

9. Casati G. Il sistema di budget. Indagine sul processo di aziendalizzazione nel SSN. RSS 1998; vol II.

10. Indicatori per il controllo dell'efficienza e dell'economicità della spesa nel SSN. Sperimentazione indicatori ospedalieri. Mediobanca 1993.

11. Wilson JD, Cambell JB. Rapporti di gestione per la direzione d'Impresa. Controllership Wilery \& Son 1981

12. Anselmi L, Volpato O. L'azienda salute. Milano: Giuffré Ed.

13. Buccoliero L. I sistemi informativi delle aziende sanitarie: fabbisogni e percorsi evolutivi verso l'a- 
dozione degli ERP. Mecosan 1998; 28.

14. Del Vecchio M. Strategia e pianificazione strategica nelle aziende sanitarie pubbliche: Un'introduzione. Mecosan 1995; 14.

15. Scheggi M. Il controllo di gestione nelle Aziende Sanitarie. Roma. Cidas 1997.

16. Zanetti $\mathrm{M}$ et al. Il medico e il management. Genova: Accademia Nazionale di Medicina 1996.

17. Del Vecchio M. Strategia e pianificazione strategica nelle aziende sanitarie pubbliche. Mecosan 17.

18. Gainnino MM. Il budget del medico ospedaliero. Ed. Medico Scientifiche 1998.

19. Dirindin N, Venes P. Elementi di economia sanitaria. Il Mulino 1999.

20. Horton R. Lancet 1998; 351, Commentary.

21. Rappini V. Il processo decisionale e gli organi coinvolti nel sistema di budget "Manuale operativo del sistema di budget nelle Aziende Sanitarie della Regione Lombardia".

22. Wagner EH. The cost-quality relationship. Do we always get what we pay for? JAMA 1994; 272 (24): 1951-2. 